\title{
Intersecting Invariant Manifolds in Spatial Restricted Three-Body Problems: Design and Optimization of Earth-to-Halo Transfers in the Sun-Earth-Moon Scenario
}

\author{
A. Zanzottera ${ }^{\mathrm{a}, \mathrm{b}, *}$, G. Mingotti ${ }^{\mathrm{a}}$, R. Castelli ${ }^{\mathrm{a}, \mathrm{c}}$, M. Dellnitz ${ }^{\mathrm{a}}$ \\ ${ }^{a}$ Institut für Industriemathematik, Universität Paderborn \\ Warburger Str. 100, 33098 Paderborn, Germany. \\ ${ }^{b}$ Dipartimento di Matematica,Politecnico di Milano \\ Piazza L. da Vinci, 20133 Milano, Italy. \\ ${ }^{c}$ BCAM - Basque Center for Applied Mathematics \\ Bizkaia Technology Park, 48160 Derio, Bizkaia, Spain.
}

\begin{abstract}
This work deals with the design of transfers connecting LEOs with halo orbits around libration points of the Earth-Moon CRTBP using impulsive maneuvers. Exploiting the coupled circular restricted three-body problem approximation, suitable first guess trajectories are derived detecting intersections between stable manifolds related to halo orbits of EM spatial CRTBP and Earth-escaping trajectories integrated in planar Sun-Earth CRTBP. The accuracy of the intersections in configuration space and the discontinuities in terms of $\Delta v$ are controlled through the box covering structure implemented in the software GAIO. Finally first guess solutions are optimized in the bicircular four-body problem and single-impulse and two-impulse transfers are presented.
\end{abstract}

Keywords: Three-body problems, Halo orbits, Invariant manifolds, Box covering, Bicircular model, Trajectory optimization.

\section{Introduction}

Halo orbits are well known three-dimensional periodic solutions around the collinear libration points of the Circular Restricted Three Body Problem (CRTBP) [38].

R.W. Farquhar was one of the firsts to recognize the applicative importance of these orbits either in Earth-Moon and Sun-Earth system [17]. Due to their location, halo orbits are useful to perform experiments and observations concerning the solar and space structure (L1-L2 libration point orbits in Sun-Earth system), to provide continuous communication between Earth and the far-side of the Moon ( L2 libration point orbits in Earth-Moon system)[16] or as parking orbits in lunar exploration missions [30]. Since 1978, when NASA launched the first halo orbit mission (ISEE3) [18], many other missions have taken advantage of the observational benefits

${ }^{*}$ Corresponding author

Email addresses: annazanz@math.upb.de (A. Zanzottera), mingotti@math.upb.de (G. Mingotti), robertoc@math.upb.de (R. Castelli), dellnitz@math.upb.de (M. Dellnitz) 
associated with these libration point orbits; among them there are the SOHO [21] mission, the Genesis [29] mission, $\mathrm{ACE}^{1}$ and the upcoming LISA Pathfinder ${ }^{2}$.

In the last decades different techniques have been developed for the design of low energy transfers in the Earth-Moon system. Some of them consider the low thrust propulsion, see for instance $[33,32,36,25,23]$ while concerning impulsive maneouvre trajectories, two are the main approaches based respectively on the CRTBP and on the Weak Stability Boundary (WSB) concept. The first one exploits the dynamics of the Earth-Moon CRTBP and in this framework different transfer from Earth to three-dimensional periodic or quasi periodic orbits have been proposed [1,31, 34, 41]. In particular in [31] transfers with three impulsive maneuvers are presented taking full advantage of lunar flyby.

In the second approach, the Earth-Moon restricted model is augmented with the perturbation of the Sun yielding a relevant reduction of the energy required for the transfer. The WSB concept was first introduced by Belbruno [3] in 1987 to design low energy, ballistic transfers to the Moon and it was applied for the rescue of the Hiten mission [8]. Since that time, the WSB theory has been deeply analyzed, see for instance $[6,19]$, and successfully applied in the design of several efficient trajectories,[11, 7, 9, 39, 12, 35].

From the perspective of the invariant manifold dynamics, a first analysis of ballistic capture transfer is done in [5] and a systematic exploration of the Tube dynamics was considered in $[28,27]$ yielding to the coupled CRTBP approximation. Basically the invariant manifolds of the periodic orbits in different circular restricted three body problems provide dynamical channels in the phase space that can be profitably exploited to accomplish various mission requirements, [22, 40, 26, 24, 13].

In this paper the coupled CRTBP approximation [28] is considered to design first guesses of low energy ballistic trajectories from a Low-Earth-Orbit (LEO) to halo orbits located around $L 2$ libration point in Earth-Moon system. The trajectory results from two stages: first the planar Sun-Earth CRTBP is adopted and the spacecraft is moved away from the LEO orbit as in the Exterior WSB transfers, then the stable invariant manifold of the target orbit in the spatial Earth-Moon CRTBP is considered for the spacecraft to be ballistically captured to the Halo orbit .

Possible connections between the two legs of the trajectory are detected on a suitable Poincaré section. Emphasis is given to the box covering technique implemented to identify in a systematic way connecting points in the phase space. The box covering, based on the software package GAIO, allows to deal easily with flows of different dimensions as well as to control the accuracy of the intersections in the configuration space.

Then, the first guess solutions obtained are optimized in the framework of the Sun-perturbed Earth-Moon bicircular four-body problem, through a direct method

\footnotetext{
${ }^{1}$ http://www.srl.caltech.edu/ACE/

${ }^{2}$ http://sci.esa.int/science-e/www/area/index.cfm?fareaid $=40$
} 
approach and a multiple shooting technique [10], [37]. Finally, trajectories with single-impulse and two-impulse maneuvers are presented and compared with results already known in literature [35].

\section{Dynamical Models}

This section outlines the dynamical systems used to study the motion of a spacecraft under the gravitational effect of three massive bodies.

\subsection{Circular Restricted Three-Body Problem}

The circular restricted three-body problem (CRTBP) [38] studies the motion of a massless particle $m_{3}$ under the gravitational influence of two main primaries, moving in circular orbits, as shown in Fig. 1(a).

In a rotating reference frame where the units of measure are normalized so that the distance between the primaries, the modulus of their angular velocity and the total mass are equal to 1 , the motion of the third body is governed by the system of equations

$$
\left\{\begin{array}{l}
\ddot{x}-2 \dot{y}=\Omega_{x} \\
\ddot{y}+2 \dot{x}=\Omega_{y} \\
\ddot{z}=\Omega_{z}
\end{array}\right.
$$

where $\Omega(x, y, z)=\frac{1}{2}\left(x^{2}+y^{2}\right)+\frac{1-\mu}{r_{1}}+\frac{\mu}{r_{2}}+\frac{1}{2} \mu(1-\mu)$ is the effective potential of the system and the subscripts denote partial derivatives. Here $\mu=m_{2} /\left(m_{1}+m_{2}\right)$ is the mass ratio of the primaries, while $r_{1}^{2}=(x+\mu)^{2}+y^{2}+z^{2}$ and $r_{2}^{2}=(x-1+\mu)^{2}+y^{2}+z^{2}$ are the distances from the spacecraft respectively to the larger and the smaller primary. Let $\phi$ be the flow associated to the system (1), so that $\phi\left(\xi, t_{i} ; t_{f}\right)$ is the solution at time $t_{f}$ leading from the initial data $\xi=(x, y, z, \dot{x}, \dot{y}, \dot{z})\left(t_{i}\right)$.

System (1) admits a first integral of motion, the Jacobi integral, defined as:

$$
C(x, y, z, \dot{x}, \dot{y}, \dot{z})=2 \Omega(x, y, z)-\left(\dot{x}^{2}+\dot{y}^{2}+\dot{z}^{2}\right)
$$

then, as $C^{*}$ varies, the family of 5-dimensional energy manifolds

$$
\mathcal{M}\left(C^{*}\right)=\left\{(x, y, z, \dot{x}, \dot{y}, \dot{z}): C(x, y, z, \dot{x}, \dot{y}, \dot{z})=C^{*}\right\}
$$

is a foliation of the 6 -dimensional phase space. For every $C^{*}$ the solution in the configuration space of the equation $C^{*}=2 \Omega(x, y, z)$ detects the zero-velocity surface, which bounds the Hill's region where the motion is possible and where it is forbidden.

The system (1) admits five equilibrium points, referred to as Lagrange points and denoted with $L i, i: 1 \ldots 5$ : three of them $L 1, L 2, L 3$ lie on the x-axis and represent collinear configurations, while $L 4, L 5$ correspond to equilateral configurations of the masses.

The topology of the Hill's region changes in correspondence to the values $C_{i}$ of the Jacobi constant relative to the libration points, allowing to open necks between different regions on the configuration space. The collinear libration points, as well as the continuous families of planar and spatial periodic orbits, respectively Lyapunov 


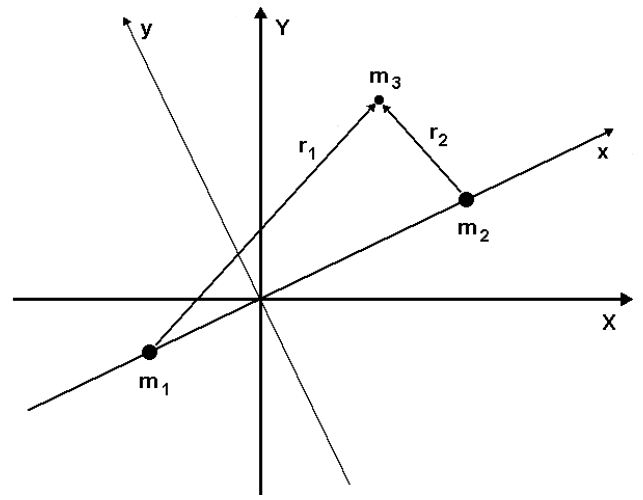

(a) Classic circular planar restricted threebody problem.

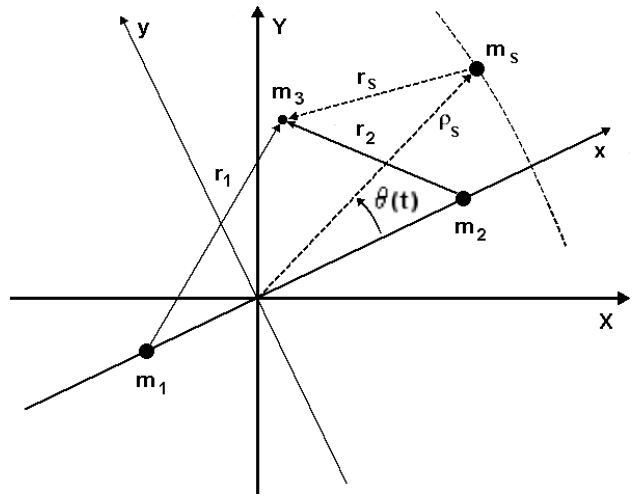

(b) Sun-perturbed Earth-Moon RTBP.

Figure 1: Mathematical models to described the physics of the problem.

and Halo orbits, exhibit a saddlexcenter type stability character. The invariant manifold structures related to these orbits act as separatrices in the energy surface and provide dynamical channels in the phase space useful to the design of energy efficient spacecraft trajectories [27].

In the following, to avoid any ambiguity between the two restricted models used in the design process, the Jacobi constant $C$, its value $C_{i}$ in correspondence of the libration points and the flow $\phi$ will be labelled with the subscripts $(\cdot)_{S E}$ and $(\cdot)_{E M}$ when they refers respectively to the planar Sun-Earth CRTBP and the spatial Earth-Moon CRTBP.

\subsection{Bicircular Restricted Four-Body Problem}

The bicircular four-body model (BCRFBP) is a restricted four-body problem where two of the primaries (the Earth and the Moon) are moving in circular orbit around their center of mass $B$ that is at the same time orbiting, together with the last mass (the Sun), around the barycenter of the system. The motion of the primaries is supposed to be co-planar and with constant angular velocity. Aiming to write the equations of motion of the BCRFBP in the EM synodical reference frame, with units of measure normalized as in the EM CRTBP, denote with $\theta(t)$ the phase of the Sun, with $\rho_{s}$ the distance between the Sun and the EM barycenter and with $r_{s}$ the Sun-spacecraft distance (see Fig. 1(b)):

$$
r_{s}^{2}=\left(x-\rho_{s} \cos \theta\right)^{2}+\left(y-\rho_{s} \sin \theta\right)^{2}+z^{2} .
$$

Then the motion of the massless particle is governed by the following system of differential equations, known also as Sun-perturbed Earth-Moon CRTBP

$$
\left\{\begin{array}{l}
\ddot{x}-2 \dot{y}=\Omega_{B x} \\
\ddot{y}+2 \dot{x}=\Omega_{B y} \\
\ddot{z}=\Omega_{B z}
\end{array}\right.
$$

where $\Omega_{B}=\Omega+\frac{m_{s}}{r_{s}}-\frac{m_{s}}{\rho_{s}^{2}}(x \cos \theta+y \sin \theta)$.

This model can be considered a good approximation of the real Sun-Earth-Moonspacecraft four-body dynamics being the eccentricities of both Earth's and Moon's 


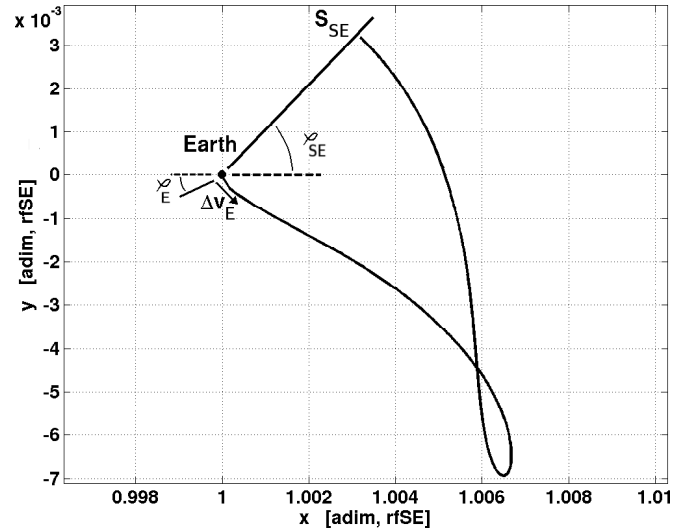

(a) Earth escape trajectory.

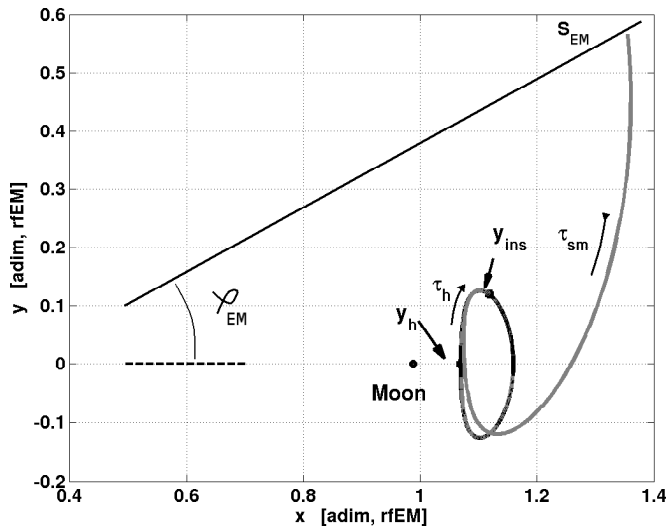

(b) Halo arrival trajectory.

Figure 2: The two stages of the Earth-to-halo trajectory design.

orbits very small (respectively 0.016 and 0.054 ) and the Moon's orbit inclined respect to the ecliptic by only $5 \mathrm{deg}$. On the other hand, it's worth noting that in the bicircular model the motion of the primaries does not solve the three-body problem. A more coherent model is the Quasi-Bicircular Problem proposed in [2].

\section{Trajectory Design}

The aim of this paper is to construct trajectories in the bicircular model, starting from a LEO and targeting a halo orbit around $L 2$ in the EM system. The design is first performed in the CRTBP and then the initial guess trajectories are optimized to be solution of the bicircular model.

The model adopted as approximation of the Sun-Earth-Moon-spacecraft restricted four-body problem is the so called Coupled CRTBP [28]. It consists in the superposition of SE and EM CRTBP: the former is used to model the motion in the first part of the mission, then when the spacecraft is enough close to Moon to benefit of its gravitational attraction, the EM CRTBP is introduced and the dynamical properties of the invariant manifold structures are exploited to fulfill the requirements of the mission.

The intersection of the two flows is analyzed on a Poincaré section allowing to detect connections between two legs of trajectory, eventually applying an impulsive maneuver, such as an out-of-plane contribution, required to obtain continuity in velocity space.

In this work different Poincaré sections will be introduced, each one defined as an hyperplane in the phase space, whose projections on the configuration space reduces to a line passing through the Earth.

\subsection{Earth Escape Stage}

The departure leg of the transfer consists in a trajectory leaving a LEO of $h_{E}=$ $167 \mathrm{~km}$ of altitude and integrated in planar SE CRTBP until the Poincaré section is reached. Any initial state $\mathbf{y}_{E}$ is identified by the angle $\varphi_{E}$, that parametrizes the 
position on the LEO with respect to a fixed direction, and the tangential impulsive maneuver $\Delta v_{E}$ provided by the launch vehicle and applied to insert the spacecraft into a translunar trajectory (see Fig. 2(a)). Denote with

$$
\mathcal{I}=\left\{\mathbf{y}_{E}\left(\varphi_{E}, \Delta v_{E}\right): 0 \leq \varphi_{E} \leq 2 \pi, \Delta v_{E} \in \mathrm{I}_{\mathrm{v}}\right\}
$$

the initial domain, where the magnitude of the initial maneuver is properly chosen in order to decrease $C_{S E}$ of the spacecraft from the initial value evaluated on the Earth parking orbit to a value in the interval $I_{C}=[3.000154,3.0024]$.

The upper bound of the interval $I_{C}$ has been chosen as the maximum value of $C_{S E}$ that allows the Moon orbit to belong to the Hill's region.

The variation of the Jacobi constant produced by a maneuver $v$ applied in the same direction of the motion is given by the formula

$$
\Delta C_{S E}=|v|^{2}+2\left|\frac{v_{t}}{r}-1\right||v| r
$$

where $v_{t}$ is the velocity of a probe orbiting on a LEO and $r$ the geocentric distance, both quantities, as well as $v$, expressed in SE dimensionless units.

Therefore, being $C_{S E}=3.07051021 \pm 3 \cdot 10^{-9}$ the Jacobi constant associated to a body orbiting on a LEO of $167 \mathrm{~km}$ of altitude, the $\Delta v_{E}$ maneuver has to be chosen in the range $\mathrm{I}_{\mathrm{v}}=[3210,3300] \mathrm{m} / \mathrm{s}$.

Once the angle $\varphi_{S E}$ between the Poincaré section and the $x$-axis is fixed, the initial states $\mathbf{y}_{E} \in \mathcal{I}$ are forward integrated. Let be denoted with

$$
P_{S E}\left(\varphi_{S E}\right)=\left\{\phi_{S E}\left(\mathbf{y}_{E}, 0 ; t\right) \cap S_{S E}\left(\varphi_{S E}\right), \mathbf{y}_{E} \in \mathcal{I}, t \in[0,1]\right\}
$$

the set of all the possible intersections with the Poincare section. Note that the cut of $\phi_{S E}\left(\mathbf{y}_{E}, 0 ; t\right)$ with the Poincaré section is not guaranteed for all the $\mathbf{y}_{E} \in \mathcal{I}$. Indeed for large enough initial maneuver, $\Delta_{v_{E}}>3260 \mathrm{~m} / \mathrm{s}$, the lobe of the Hill's region containing the Earth joins the internal and external regions, thus some of the trajectories may escape the Earth lobe before intersecting the section. This occurs in particular for $\Delta v_{E}>3270 \mathrm{~m} / \mathrm{s}$, when the stable manifold emanating from the two Lyapunov orbits invest the Earth. In that case a small change in the initial position on the Earth parking orbit produces very different results: some trajectories will transit through the Lyapunov orbits, others will match the Poincaré section in far away points, being the amount of the twist produced on the trajectory very sensitive with respect the initial position, [27].

\subsection{Halo Capture Stage}

In the second phase of the design the spatial EM CRTBP is considered to govern the motion of the spacecraft and the invariant manifold structure associated to the periodic orbits is exploited to fulfill the mission requirements. Indeed, concerning the problem to target a halo orbit, once the spacecraft is placed on the stable manifold it will fly for free along the manifold until, theoretically after an infinite time, it will be inserted into the periodic orbit. 
Let $\lambda_{2}$ be used to denote the target halo orbit and $\mathbf{y}_{h}$ its inner $x$-axis crossing. To every point $\mathbf{y}_{\text {ins }}$ on the halo, in the following referred as insertion point, is associated the time $\tau_{h} \in[0, T]$ solution of $\mathbf{y}_{\text {ins }}=\phi_{E M}\left(\mathbf{y}_{h}, 0 ; \tau_{h}\right)$, being $T$ the period of the halo. As depicted in Fig. 2(b), any point $\mathbf{y}_{\text {ins }}$ is then slightly perturbed into $\tilde{\mathbf{y}}_{\text {ins }}=\mathbf{y}_{\text {ins }}+\varepsilon \mathbf{v}_{s}$, where the stable direction $\mathbf{v}_{s}$ is provided by the monodromy matrix, and each state $\tilde{\mathbf{y}}_{\text {ins }}$ is backwards integrated. The stable manifold $W^{s}\left(\lambda_{2}\right)$ is defined as

$$
W^{s}\left(\lambda_{2}\right)=\bigcup_{\tilde{\mathbf{y}}_{\text {ins }}}\left\{\mathbf{y}_{s m}=\phi\left(\tilde{\mathbf{y}}_{\text {ins }}, 0 ; \tau_{s m}\right), \forall \tau_{s m}<0\right\}
$$

and it consists in a two-dimensional cylinder parametrized by $\tau_{h} \in[0, T]$ and $\tau_{s m} \in$ $(-\infty, 0)$. By construction, once the spacecraft is placed onto any point of the stable manifold, the couple $\left(\tau_{h}, \tau_{s m}\right)$ univocally determine how long the probe needs to fly to reach the target orbit and which is the particular location of the insertion point.

In analogy with the notation adopted in the previous section, for a choice of the angle $\varphi_{E M}$ between the Poincaré section $S_{E M}\left(\varphi_{E M}\right)$ and the $x$-axis, define

$$
P_{E M}\left(\varphi_{E M}\right)=W^{s}\left(\lambda_{2}\right) \cap S_{E M}\left(\varphi_{E M}\right),
$$

in the following denoted as Poincaré map, the first intersection of the invariant tube with the section. Since only the first intersection is taken into account, the Poincaré map is topologically equivalent to a circle, still parametrized by $\tau_{h}$.

\subsection{Trajectory connection}

The last stage of the design process consists in gluing together, with the aid of a impulsive maneuver, a Earth escaping orbit and a halo captured trajectory yielding a complete Earth-to-halo transfer. The design of the whole Earth-to-halo trajectory restricts to the selection of pairs of points $\mathbf{y}_{S E} \in P_{S E}$ and $\mathbf{y}_{E M} \in P_{E M}$. The necessary condition to identify feasible trajectories is the coincidence, at least in configuration space, of the points $\mathbf{y}_{S E}$ and $\mathbf{y}_{E M}$. That first requires the two surfaces of section $S_{S E}$ and $S_{E M}$ to project on the same line in the $(x, y)$ plane. In terms of mutual position of the primaries, it means that the Earth-Moon line must be tilted on the angle $\beta=\varphi_{S E}-\varphi_{E M}$ respect to the Sun-Earth line at the instant the spacecraft is on the section. Moreover, since the Poincaré map $P_{S E}$ lives in the $\{z=0\}$ plane, the search of possible intersections is restricted to those points in $P_{E M}$ with zero $z$-coordinate. Concerning the $\Delta V$ maneuver, since the spatial flow is never tangential to the $\{z=0\}$ plane (or it restricts to the planar motion), even if it is possible to achieve satisfying intersections in configuration space between $P_{S E}$ and $P_{E M} \cap\{z=0\}$, the out-of-plane component of the velocity maneuver can never be avoided.

Candidate transfer point pairs are sought by varying the design parameters $\varphi_{S E}$, $\varphi_{E M}, \Delta v_{E}, \varphi_{E}, \mathbf{y}_{\text {ins }}, C_{E M}$ in their range of definition and an efficient and systematic search is numerically performed by means of a covering algorithm based on the box structures implemented within the software package GAIO (see the next subsection). 


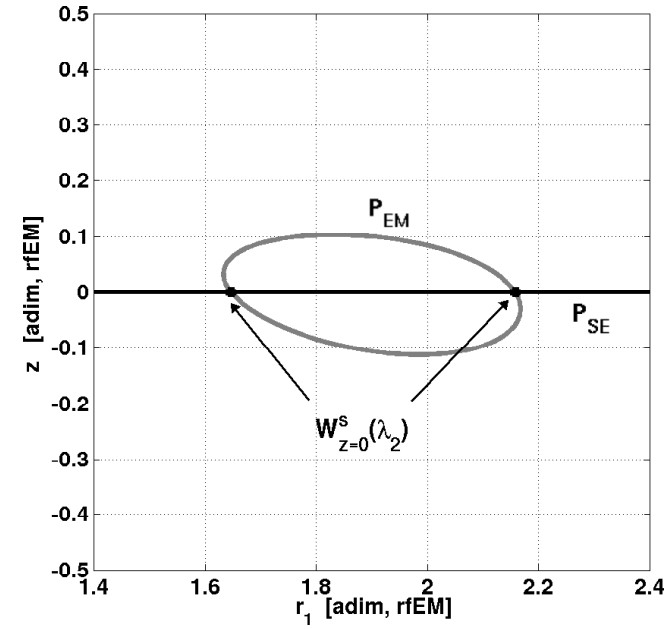

(a) $P_{S E} \cap P_{E M}$ in the $r_{1}, z$ coordinates.

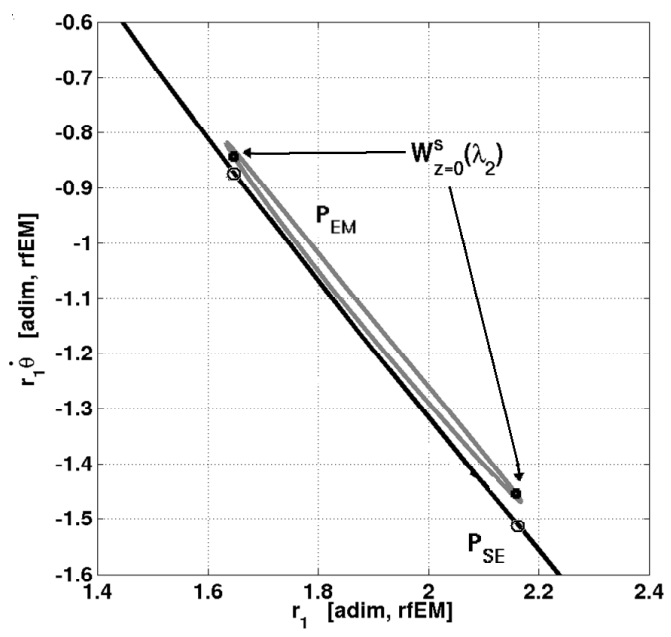

(c) $P_{S E} \cap P_{E M}$ in the $r_{1}, r_{1} \dot{\theta}$ coordinates.

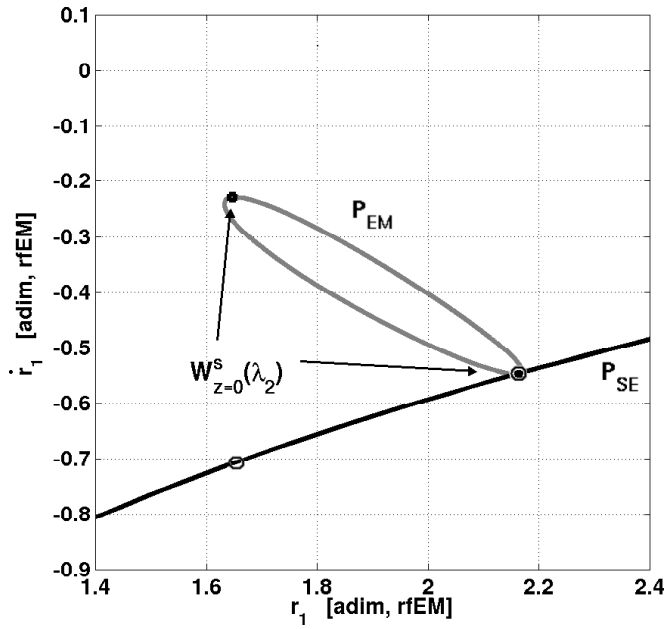

(b) $P_{S E} \cap P_{E M}$ in the $r_{1}, \dot{r}_{1}$ coordinates.

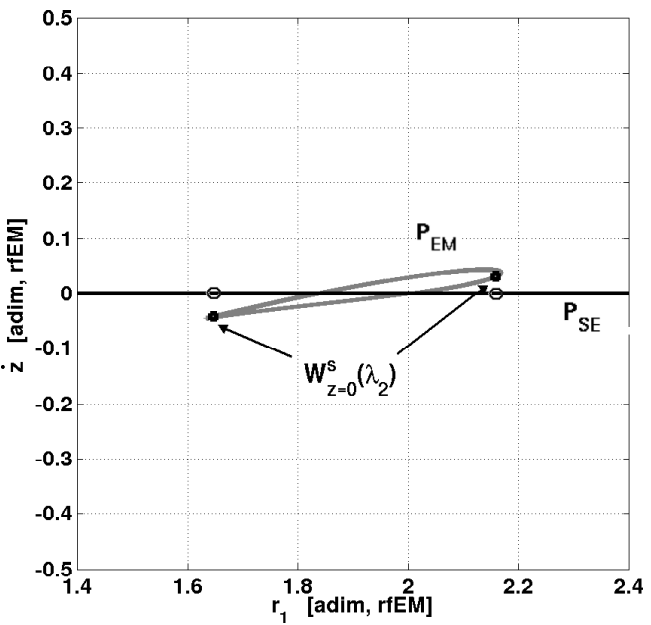

(d) $P_{S E} \cap P_{E M}$ in the $r_{1}, \dot{z}$ coordinates.

Figure 3: Transfer point definition on suitable Poincaré maps.

In the numerical simulations presented, in order to have a better visualization of the selection of the transfer points, taking full advantage of the the geometry of the maps, a system of cylindrical coordinates $\left(r_{1}, \theta, z, \dot{r}_{1}, r_{1} \dot{\theta}, \dot{z}\right)$ is adopted. Here $\left(r_{1}, \theta\right)$ denotes the polar coordinates in the plane of the primaries with the Earth as center and $\theta=0$ the Earth-Moon line, while $z$ the out-of-plane component. Fig. 3 depicts the Poincaré maps $P_{E M}, P_{S E}$ for a choice of $\varphi_{S E}=\frac{3}{4} \pi, \varphi_{E M}=\frac{\pi}{4}$, $\Delta v_{E}=3280 \mathrm{~m} / \mathrm{s}, C_{E M}=3.1495576$, while $\varphi_{E}$ varies in $[0,2 \pi]$ and $\mathbf{y}_{\text {ins }}$ along the whole halo. In particular the subfigure Fig. 3(a) shows the projection into the $\left(r_{1}, z\right)$ plane, while the remaining subfigures concern the velocity components. The grey ring and black line stand respectively for $P_{E M}$ and $P_{S E}$ and the black dots and the circles represents the intersections of the two maps in configuration space.

\subsection{Box Covering Technique}

GAIO (Global Analysis of Invariant Objects) is a set oriented software package based on subdivision methods, with a searchable tree structured architecture, de- 


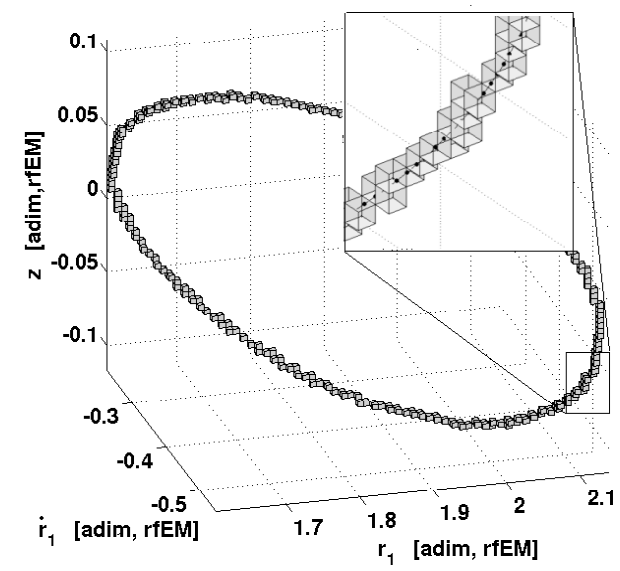

(a) Box covering of $P_{E M}$

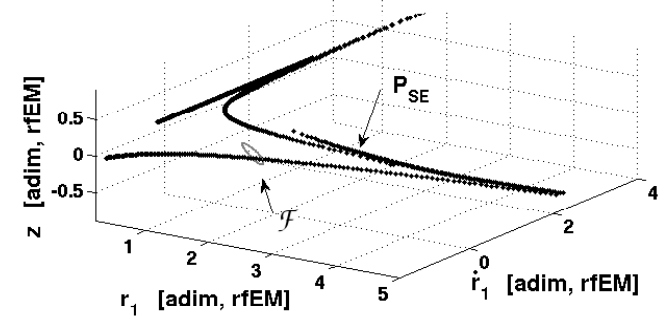

(b) $P_{S E}$ and the covering of $P_{E M}$

Figure 4: Left: the black dots are the points of $P_{E M}$ covered by the box collection $\mathcal{F}$. Right: $\mathcal{F}$ and $P_{S E}$ plotted in $\left(r_{1}, \dot{r}_{1}, z\right)$ cylindrical coordinates.

signed to study the global structure of a given dynamical system [14]. Although the numerical methods provided by GAIO could be adopted in all the stages of the mission design, showing versatility and efficiency [13], in this work only the basic box structures and the routines for their management have been exploited.

A box $\mathcal{B}(C, R)$ is a $\mathrm{N}$-dimensional rectangle, univocally identified by a center $C=\left(C_{1}, \cdots, C_{N}\right) \in \mathbb{R}^{N}$ and a vector of radii $R=\left(r_{1}, \cdots, r_{n}\right) \in \mathbb{R}^{N}$, defined as

$$
\mathcal{B}(C, R)=\bigcap_{i=1}^{N}\left\{\left(x_{1}, x_{2}, \ldots x_{N}\right) \in \mathbb{R}^{N}:\left|x_{i}-C_{i}\right|<r_{i}\right\} .
$$

Starting from an initial box $\mathcal{B}_{0}$ containing $P_{E M}$, a multiple subdivision process is carried out to create families $\mathcal{F}_{k}$ of smaller boxes $\left\{\mathcal{B}_{k}\right\}$ with the property to cover $\mathcal{B}_{0}$, i.e. $\cup \mathcal{B}_{k}=\mathcal{B}_{0}$. In the $k$-th subdivision step each rectangle $\mathcal{B}(C, R)$ of the existing collection $\mathcal{F}_{k}$ is subdivided with respect to the $j$-th coordinate, where $j$ can vary cyclically or can be chosen by the user. That means that passing from $\mathcal{F}_{k-1}$ to $\mathcal{F}_{k}$ the radius $r_{j}$ is halved, keeping $r_{i}, i \neq j$ unchanged, and the number of boxes increases twofold. Once the radii of the boxes in $\mathcal{F}_{k}$ reach a prescribed size, the Poincaré map is inserted: only those boxes in $\mathcal{F}_{k}$ with non empty intersection with $P_{E M}$ are stored, otherwise removed. Denote with box covering of the map $P_{E M}$ the collection $\mathcal{F}$ of the remaining boxes, see Fig. 4(a). Note that the freedom of choosing the radii along which to perform the subdivision, implies that the accuracy of the covering could be set independently in each dimension. Then possible connections are sought looking at the set $E=\mathcal{F} \cap P_{S E}$, see Fig. 4(b). Since $P_{S E}$ lies on the $\{z=0\}$ plane at most two boxes of $\mathcal{F}$, say $B_{1}, B_{2}$, are involved in the above intersection. Denoting with $E_{i}$ the subset of $E$ whose elements are in $B_{i}$ and with $M_{i}$ the subset of $P_{E M}$ covered by $B_{i}(i=1,2)$, the preliminary set of transfer point pairs is defined as

$$
T=\bigcup_{i=1,2}\left\{\left(\mathbf{y}_{S E}, \mathbf{y}_{E M}\right): \mathbf{y}_{S E} \in M_{i}, \mathbf{y}_{E M} \in E_{i}\right\} .
$$


Finally, in $T$ the pair which minimizes the distance in velocity space is selected as candidate transfer point pair. In this work, the necessary feasibility condition is considered satisfied once the covering $\mathcal{F}$ is at least $10^{-4}$ (in EM units) accurate in configuration space.

\section{Trajectory Optimization}

This section gives firstly a brief introduction of the trajectory optimization approach used in this work, then formulates in details the minimization problems later solved.

Once feasible and efficient first guess solutions are achieved, combining the two legs of the transfer, an optimization problem is stated. A given objective function is minimized taking into account the dynamic of the process.

The dynamical model used to consider the gravitational attractions of all the celestial bodies involved in the design process (i.e. the Sun, the Earth, and the Moon) is the spatial BCRFBP described by (4) (written in an autonomous fashion) with the adding of the term that corresponds to the rotation of the Sun:

$$
\left\{\begin{array}{l}
\ddot{x}-2 \dot{y}=\Omega_{B x} \\
\ddot{y}+2 \dot{x}=\Omega_{B y} \\
\ddot{z}=\Omega_{B z} \\
\dot{\theta}=\omega_{s}
\end{array}\right.
$$

According to the formalism proposed by Betts [10], the BCRFBP described by (5) is written in the first-order form

$$
\begin{aligned}
\dot{x} & =v_{x} \\
\dot{y} & =v_{y} \\
\dot{z} & =v_{z} \\
\dot{v}_{x} & =2 v_{y}+\Omega_{B x} \\
\dot{v}_{y} & =-2 v_{x}+\Omega_{B y} \\
\dot{v}_{z} & =\Omega_{B z} \\
\dot{\theta} & =\omega_{s}
\end{aligned}
$$

with $v_{x}=\dot{x}, v_{y}=\dot{y}$ and $v_{z}=\dot{z}$. In a compact explicit form, system (6) reads

$$
\dot{\mathbf{y}}=\mathbf{f}[\mathbf{y}(t), \mathbf{p}, t],
$$

where $\mathbf{f}$ stands for the vector field and $\mathbf{y}=\{x, y, z, \dot{x}, \dot{y}, \dot{z}, \theta\}^{\top}$ is the state vector. The aim is finding $\mathbf{y}=\mathbf{y}(t), t \in\left[t_{i}, t_{f}\right]$, that minimizes a prescribed scalar performance index or objective function

$$
J=J(\mathbf{y}, \mathbf{p}, t),
$$

while satisfying certain mission constraints. These constraints are represented by the two boundary conditions, defined at the end points of the optimization problem, and by the inequality conditions, defined along the whole arc. These last quantities 
are derived specifically for the mission investigated. Moreover, $\mathbf{p}$ stands for a vector which brings together some parameters useful for the optimization process.

The optimization problem, OP, is then transcribed into a nonlinear programming problem, NLP, using a direct approach. This method, although suboptimal, generally shows robustness and versatility, and does not require explicit derivation of the necessary conditions of optimality. Moreover, direct approaches offer higher computational efficiency and are less sensitive to variation of the first guess solutions [10]. Furthermore, a multiple shooting scheme is implemented. With this strategy, the BCRFBP dynamics presented by (5) is forward integrated within $N-1$ intervals (in which $\left[t_{i}, t_{f}\right]$ is uniformly split), i.e. the time domain is divided in the form $t_{i}=t_{1}<\cdots<t_{N}=t_{f}$, and the solution is discretized over the $N$ grid nodes. The continuity of position and velocity is imposed at their ends [15], in the form of defects $\boldsymbol{\eta}_{j}=\overline{\mathbf{y}}_{j}-\mathbf{y}_{j+1}=0$, for $j=1, \ldots, N-1$. The quantity $\overline{\mathbf{y}}_{j}$ stands for the result of the integration, i.e. $\overline{\mathbf{y}}_{j}=\phi\left(\mathbf{y}_{j}, t\right), t_{j} \leq t_{j+1}$. The algorithm computes the value of the states at mesh points, satisfying both boundary and path constraints, and minimizing the performance index.

Dynamics described by (5) are highly nonlinear and, in general, lead to chaotic orbits. In order to find accurate optimal solutions without excessively increasing the computational burden, an adaptive nonuniform time grid has been implemented. Thus, when the trajectory is close to either the Earth or the Moon the grid is offline refined, whereas in the intermediate phase, where a weak vector field governs the motion of the spacecraft, a coarse grid is used. The optimal solution found is assessed a posteriori by forward integrating the optimal initial condition with a Runge-Kutta $8^{\text {th }}$ order scheme.

As far as it concerns the optimization algorithms, the Fortran package for largescale NLP, called SNOPT, has been used [20]. SNOPT is a general-purpose system for solving optimization problems involving many variables and constraints. It minimizes a linear or nonlinear function subject to bounds on the variables and sparse linear or nonlinear constraints. It is suitable for large-scale linear and quadratic programming and for linearly constrained optimization, as well as for general nonlinear programs. Moreover, the solutions found are locally optimal, and ideally any nonlinear functions should be smooth. Unknown gradients are estimated by finite differences. SNOPT uses a sequential quadratic programming (SQP) algorithm that obtains search directions from a sequence of quadratic programming subproblems. Each QP subproblem minimizes a quadratic model of a certain Lagrangian function subject to a linearization of the constraints. An augmented Lagrangian merit function is reduced along each search direction to ensure convergence from any starting point.

\subsection{Two-Impulse Problem Statement}

In this section, the approach previously described is exploited to obtain optimal transfers with two-impulsive maneuvers. According to the NLP formalism recalled, the variable vector is

$$
\mathbf{x}=\left\{\mathbf{y}_{1}, \ldots, \mathbf{y}_{N}, t_{1}, t_{N}\right\}^{\top}
$$


The initial conditions read:

$$
\begin{aligned}
& \boldsymbol{\psi}_{i}\left(\mathbf{y}_{1}, t_{1}\right):= \\
& \left\{\begin{array}{l}
\left(x_{1}+\mu\right)^{2}+y_{1}^{2}+z_{1}^{2}-r_{i}^{2}=0 \\
\left(x_{1}+\mu\right)\left(\dot{x}_{1}-y_{1}\right)+y_{1}\left(\dot{y}_{1}+x_{1}+\mu\right)+z_{1} \dot{z}_{1}=0,
\end{array}\right.
\end{aligned}
$$

which force the first $\mathbf{y}_{1}$ state of the transfer to belong to a circular orbit of radius $r_{i}=R_{E}+h_{E}$, where $R_{E}$ and $h_{E}$ stand for the Earth radius and the orbit altitude on the Earth, respectively. The transfer ends when the spacecraft flies on the halo orbits stable manifold, which is defined thanks to two parameters, as shown in Fig. 1(b). In details, only the continuity in terms of position is imposed (and not velocity), so that the final condition reads

$$
\boldsymbol{\psi}_{f}=\overline{\mathbf{y}}_{N}-\overline{\mathbf{y}}_{s m}=0
$$

where it is worth noting that $\overline{\mathbf{y}}_{N}=\left\{x_{N}, y_{N}, z_{N}\right\}^{\top}$ and $\overline{\mathbf{y}}_{s m}=\left\{x_{s m}, y_{s m}, z_{s m}\right\}^{\top}$. This means that, after the initial impulsive maneuver, a second one is required to inject the spacecraft onto the stable manifold that takes it ballistically to the final halo orbit associated.

The nonlinear equality constraint vector, made up of the boundary conditions and the ones representing the dynamics, is therefore written as follows:

$$
\mathbf{c}(\mathbf{x})=\left\{\boldsymbol{\psi}_{i}, \boldsymbol{\eta}_{1}, \ldots, \boldsymbol{\eta}_{N-1}, \boldsymbol{\psi}_{f}\right\}^{\top} .
$$

Moreover, aiming at avoiding the collision with the two primaries, the following inequality constraints are imposed:

$$
\begin{aligned}
& \mathbf{\Psi}_{j}^{c}\left(\mathbf{y}_{j}\right):=\left\{\begin{array}{l}
R_{E}^{2}-\left(x_{j}+\mu\right)^{2}-y_{j}^{2}-z_{j}^{2} \leq 0 \\
R_{M}^{2}-\left(x_{j}-1+\mu\right)^{2}-y_{j}^{2}-z_{j}^{2} \leq 0,
\end{array}\right. \\
& j=2, \ldots, N-1 .
\end{aligned}
$$

Finally, the flight time is searched to be positive, i.e.

$$
\Psi^{t}=t_{1}-t_{N} \leq 0
$$

The complete inequality constraint vector therefore reads:

$$
\mathbf{g}(\mathbf{x})=\left\{\boldsymbol{\Psi}_{2}^{c}, \ldots, \boldsymbol{\Psi}_{N-1}^{c}, \boldsymbol{\Psi}^{t}\right\}^{\top} .
$$

As for the performance index to minimize, this is a scalar that represents the two velocity variations at the beginning and at the final node of the transfer, i.e. $J(\mathbf{x})=\Delta v_{1}+\Delta v_{N}$. In details,

$$
\Delta v_{1}=\sqrt{\left(\dot{x}_{1}-y_{1}\right)^{2}+\left(\dot{y}_{1}+x_{1}+\mu\right)^{2}+\left(z_{1}\right)^{2}}-v_{i},
$$

assuming $v_{i}=\sqrt{(1-\mu) / r_{i}}$ as the velocity along the initial circular parking orbit, and

$$
\Delta v_{N}=\sqrt{\left(\dot{x}_{N}-\dot{x}_{s m}\right)^{2}+\left(\dot{y}_{N}-\dot{y}_{s m}\right)^{2}+\left(\dot{z}_{N}-\dot{z}_{s m}\right)^{2}},
$$


which represents the discontinuity in terms of velocity between the translunar trajectory and the stable manifold related to the final halo.

In summary, the NLP problem for the two-impulse transfers is formulated as follows:

$$
\begin{aligned}
& \min J(\mathbf{x}) \text { subject to } \mathbf{c}(\mathbf{x})=0 \text {, } \\
& \mathrm{x} \quad \mathrm{g}(\mathrm{x}) \leq 0 \text {. }
\end{aligned}
$$

\subsection{Single-Impulse Problem Statement}

As for the single-impulse trajectories, the variable vector is stated as follows:

$$
\mathbf{x}=\left\{\mathbf{y}_{1}, \ldots, \mathbf{y}_{N}, \mathbf{p}, t_{1}, t_{N}\right\}^{\top},
$$

where $\mathbf{p}=\left\{\tau_{h}, \tau_{s m}\right\}$, which is made up of two optimization parameters useful to describe the final condition of the transfer (see Fig. 1(b)).

The equality constraint vector is defined, as in the previous paragraph, by (12) with the exception of the final condition: in this case, at the final point of trajectory optimization, the whole dynamical state is forced to be equal to the one associated to the stable manifold (position and velocity):

$$
\boldsymbol{\psi}_{f}=\mathbf{y}_{N}-\mathbf{y}_{s m}=0
$$

where $\mathbf{y}_{N}=\left\{x_{N}, y_{N}, z_{N}, \dot{x}_{N}, \dot{y}_{N}, \dot{z}_{N}\right\}^{\top}$ and $\mathbf{y}_{s m}=\left\{x_{s m}, y_{s m}, z_{s m}, \dot{x}_{s m}, \dot{y}_{s m}, \dot{z}_{s m}\right\}^{\top}$. This means that, after the initial impulsive maneuver, the spacecraft flies ballistically (with the dynamics described by the BCRFBP of (5)) to the final selected halo orbit, with no other corrections.

In details, a generic point $\mathbf{y}_{s m}$ on the stable manifold is defined as described in section 3.2. Moreover, also the inequality constraint vector is defined in the same way as in the two-impulse scenario.

Dealing with the objective index to minimize, this is made up of only the initial velocity variation, i.e. the magnitude of the translunar insertion maneuver, i.e. $J(\mathbf{x})=\Delta v_{1}$, where

$$
\Delta v_{1}=\sqrt{\left(\dot{x}_{1}-y_{1}\right)^{2}+\left(\dot{y}_{1}+x_{1}+\mu\right)^{2}+\left(z_{1}\right)^{2}}-v_{i},
$$

assuming once again $v_{i}=\sqrt{(1-\mu) / r_{i}}$ as the velocity of the initial circular orbit.

Finally, the NLP problem for the low-energy transfers is formulated in the same fashion as proposed by (18) at the end of the previous section.

\section{Optimized Transfer Solutions}

In this section the transfers to halos obtained solving the optimization process are presented. In the previous two sections, two families of trajectories are discussed, according to the number of impulsive maneuvers that are allowed. In the following, the optimized solutions are proposed in terms of some relevant performance parameters. 


\subsection{Trajectories to Halos}

Optimal two-impulse and single-impulse solutions are presented. These transfers start from a circular parking orbit at an altitude of $h_{E}=167 \mathrm{~km}$ around the Earth, and reach a halo orbit around $L 2$, with an out-of-plane amplitude of $A_{z}=8000 \mathrm{~km}$. The results are shown in Tab. 1 as follows: sol.1.1 and sol.1.2 correspond to twoimpulse low energy transfers, while solutions sol.2.1 and sol.2.2 represent singleimpulse low energy transfers. Then, solutions below the line are some reference impulsive transfers found in literature.

More in details, Table 1 is so structured: the second column $\Delta v_{i}$ stands for the initial impulsive maneuver that inserts the spacecraft onto the translunar trajectory. The third column $\Delta v_{f}$ represents the final impulsive maneuver that permits the insertion of the spacecraft onto the stable manifold related to the target halo. This latter maneuver is present only for the two-impulse trajectories. The fourth column $\Delta v_{t}$ represents the overall amount of impulsive maneuvers necessary to complete the Earth-to-halo transfers. Finally, the last column on the right stands for the whole transfer time.

An analysis of the table shows that the single-impulse sol.2.1 (see Fig. 5(a)) offers the lowest value of the overall impulsive maneuvers (see $\Delta v_{t}$ ). This happens because the first guess solution exploits deeply the dynamics of the RTBPs where it is designed, and later of the Earth-Moon BCRFBP where it is optimized. Moreover, this trajectory takes explicitly advantage of the initial lunar flyby. The latter can be seen as a kind of aid in the translunar orbit insertion, as it reduces the $\Delta v_{i}$ required for that maneuver. The lunar flyby performs a change of plane of the translunar trajectory that allows the insertion of the spacecraft onto the three-dimensional halo stable manifold without any other maneuver. Summarizing, the single-impulse trajectory corresponding to sol.2.1, acknowledges these remarks, as it shows the lowest global $\Delta v_{t}=3161 \mathrm{~m} / \mathrm{s}$ (with travel time $\Delta t=98$ days).

Table 1: Two-impulse and single-impulse low energy transfers to halos around $L 2$. Comparison we a set of impulsive reference solutions found in literature is also reported $([35,31])$.

\begin{tabular}{lcccc} 
Type & $\Delta v_{i}[\mathrm{~m} / \mathrm{s}]$ & $\Delta v_{f}[\mathrm{~m} / \mathrm{s}]$ & $\Delta v_{t}[\mathrm{~m} / \mathrm{s}]$ & $\Delta t[$ days $]$ \\
\hline sol.1.1 & 3110 & 214 & 3324 & 97 \\
sol.1.2 & 3150 & 228 & 3378 & 120 \\
sol.2.1 & 3161 & 0 & 3161 & 98 \\
sol.2.2 & 3201 & 0 & 3201 & 126 \\
\hline Park. & 3132 & 618 & 3750 & - \\
Park. & 3235 & 0 & 3235 & - \\
Mingtao & - & - & 3480 & 17 \\
\hline
\end{tabular}




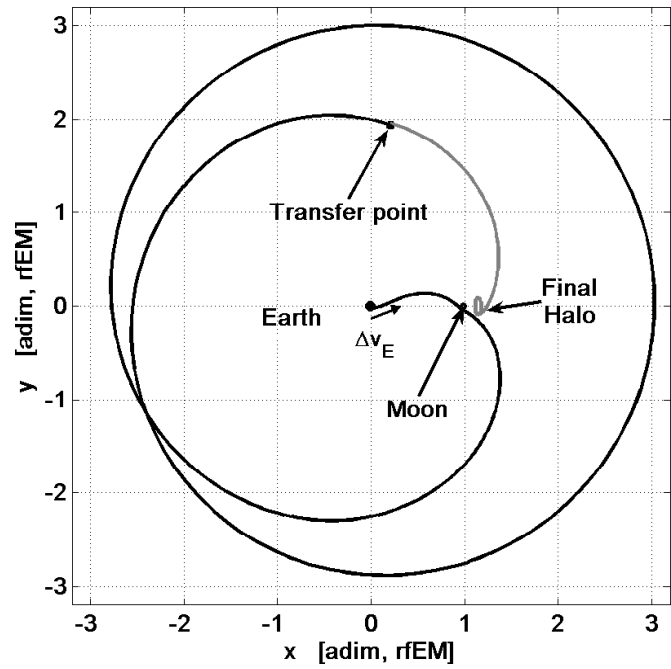

(a) Trajectory in Earth-Moon rotating frame.

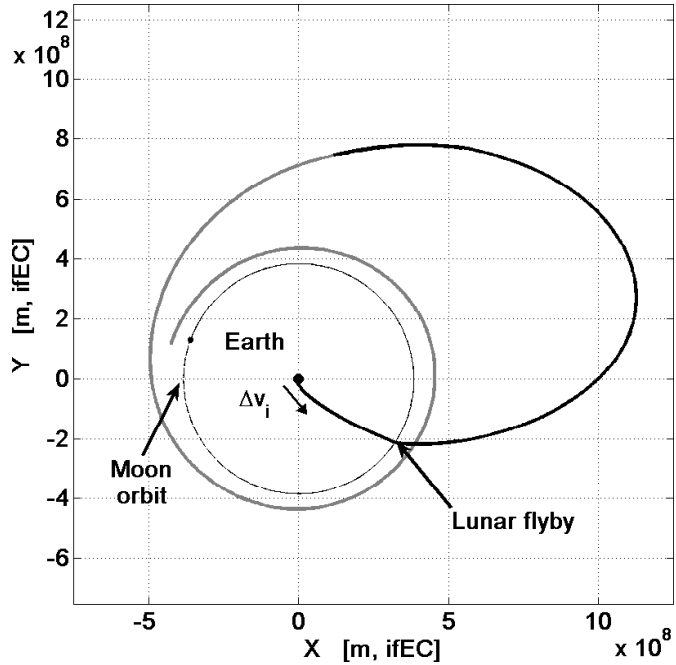

(b) Trajectory in Earth-centered inertial frame.

Figure 5: Optimized trajectory corresponding to sol.2.1 of table 1.

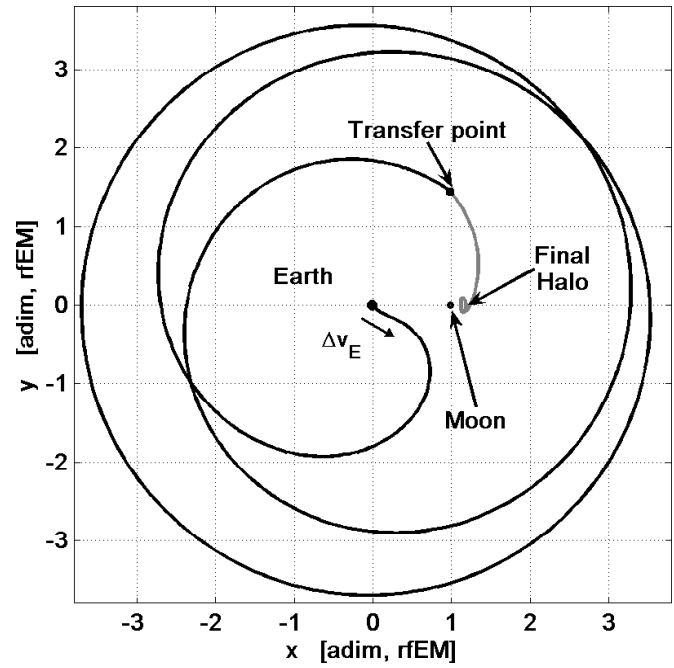

(a) Trajectory in Earth-Moon rotating frame.

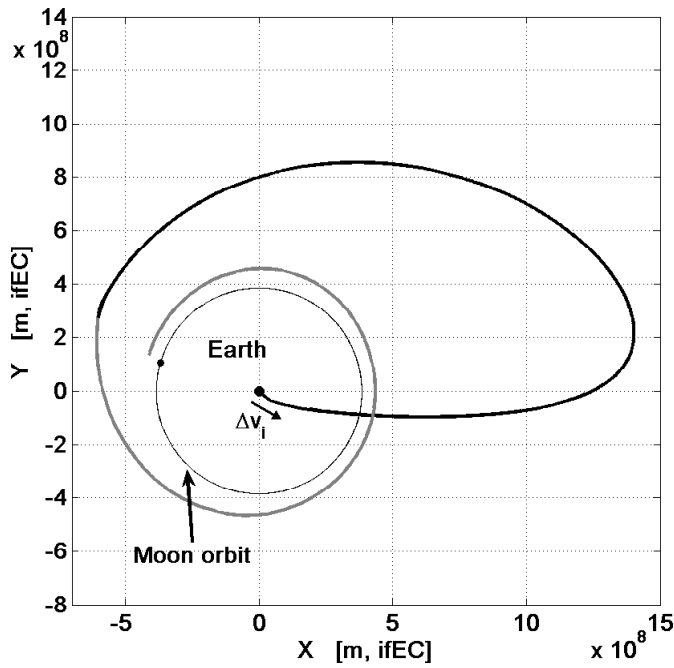

(b) Trajectory in Earth-centered inertial frame.

Figure 6: Optimized trajectory corresponding to sol.2.2 of table 1.

\section{Final Remarks}

This paper has investigated a technique to design two-impulse and single-impulse low energy transfers. They revealed to be efficient, both in terms of impulsive maneuvers and flight time. The optimization approach resulted robust and versatile, and the obtained solutions have been a posteriori validated by means of a Runge-Kutta $8^{\text {th }}$ order scheme. Moreover, through the box covering technique, an immediate definition of the transit points in the phase space was possible, allowing to formalize a systematic method to intersect three-dimensional manifolds. 


\section{Acknowledgements}

This work has been supported by the Marie Curie Actions Research and Training Network AstroNet, Contract Grant No. MCRTN-CT-2006-035151.

\section{References}

[1] E.M. Alessi, G. Gómez and J. Masdemont. Two-manoeuvres transfers between LEOs and Lissajous orbits in the Earth-Moon system. Advances in Space Research, 45(10): 1276-1291, 2010.

[2] M.A. Andreu. The quasi-bicircular problem. Phd Thesis, Departament de Matemàtica Aplicada i Anàlisi, Universitat de Barcelona, 1998.

[3] E. Belbruno. Lunar capture orbits, a method for constructing EarthMoon trajectories and the lunar GAS mission. In: Proceedings of AIAA/DGLR/JSASS Inter. Propl. Conf. AIAA Paper No. 87-1054, 1987

[4] E.A. Belbruno and J.K. Miller. A ballistic lunar capture trajectory for Japanese spacecraft Hiten. JPL IOM, 312: 90-94, 1990.

[5] E.A. Belbruno. The Dynamical Mechanism of Ballistic Lunar Capture Transfers in the Four-Body Problem from the Perspective of Invariant Manifolds and Hill'S Regions. Technical Report, Centre de Recerca Matematica, Barcelona, Spain, 1994.

[6] E.A. Belbruno. Capture Dynamics and Chaotic Motions in Celestial Mechanics. Princeton University Press, Princeton 2004.

[7] E.A. Belbruno and J.P. Carrico. Calculation of weak stability boundary ballistic lunar transfer trajectories. In AIAA/AAS Conference, 2000.

[8] E.A. Belbruno and J.K. Miller, Sun-Perturbed Earth-to-Moon Transfers with Ballistic Capture. Journal of Guidance Control and Dynamics, 16: 770-775, 1993.

[9] M. Belló, F. Graziani, P. Teofilato, C. Circi, M. Portfilio and M. Hechler, A systematic analysis on weak stability boundary transfers to the moon. 51st International Astronautical Congress, 2000.

[10] J.T. Betts. Survey of Numerical Methods for Trajectory Optimization. Journal of Guidance control and dynamics, 21(2): 193-207, 1998.

[11] C. Circi, P. Teofilato. On the dynamics of weak stability boundary lunar transfers. Celestial Mechanics and Dynamical Astronomy, 79: 41-72, 2001.

[12] C. Circi and P. Teofilatto. Weak stability boundary trajectories for the deployment of lunar spacecraft constellations. Celestial Mechanics and Dynamical Astronomy, 95: 371-390, 2006. 
[13] M. Dellnitz, O. Junge, M. Post, and B. Thiere. On Target for Venus: Set Oriented Computation of Energy Efficient Low Thrust Trajectories. Celestial Mechanics and Dynamical Astronomy, 95: 357-370, 2006.

[14] M. Dellnitz and O. Junge. Set Oriented Numerical Methods for Dynamical Systems. Handbook of dynamical systems, 2(1): 900, 2002.

[15] P.J. Enright and B.A. Conway. Discrete Approximations to Optimal Trajectories Using Direct Transcription and Nonlinear Programming. Journal of Guidance Control and Dynamics, 15: 94-1002, 1992.

[16] R.W. Farquhar. Future Missions for Libration-Point Satellites. Astronautics and Aeronautics, 7: 52-56, 1969.

[17] R.W. Farquhar. The control and use of Libration-Point Satellites. Astronautics and Aeronautics, 1968.

[18] R.W. Farquhar, D.P. Muhonen, C. Newman, and H. Heuberger. The First Libration Point Satellite, Mission Overview and Flight History. In AIAA/AAS Conference, 1979.

[19] F. Garcia and G. Gómez. A note on weak stability boundaries. Celestial Mechanics and Dynamical Astronomy, 97(2): 87-100, 2007.

[20] P.E. Gill, W. Murray and M.A. Saunders. Users Guide for SNOPT 5.3: A Fortran Package for Large-Scale Nonlinear Programming. Boeing Information and Support Services, 1998.

[21] G. Gómez, J. Masdemont, and J.M. Mondelo. A Dynamical System Approach for the Analysis of the SOHO Mission. In Third International Symposium on Spacecraft Flight Dynamics, European Space Agency, Darmstadt, Germany, 449-454, 1991.

[22] G. Gomez, W. S. Koon, M. W. Lo, J. E. Marsden, J. Masdemont and S. D. Ross Connecting orbits and invariant manifolds in the spatial restricted three-body problem Nonlinearity, 17(5): 1571-1606, 2004

[23] A.L. Herman and B.A. Conway. Optimal, Low-Thrust, EarthMoon Orbit Transfer. Journal of Guidance, Control, and Dynamics, 21(1): 141-147, 1998.

[24] K.C. Howell, B.T. Barden, and M.W. Lo. Application of Dynamical Systems Theory to Trajectory Design for a Libration Point Mission. Journal of Astronautical Sciences, 45(2): 161-178, 1997.

[25] C.A. Kluever and B.L Pierson. Optimal low-thrust three-dimensional Earthmoon trajectories. Journal of Guidance Control Dynamics, 18(4): 830-837, 1995. 
[26] W.S. Koon, M.W. Lo, J.E. Marsden, and S.D. Ross. Constructing a Low Energy Transfer Between Jovian Moons. Contemporary Mathematics, 292: 129-146, 2002.

[27] W.S. Koon, M.W. Lo, J.E. Marsden, and S.D. Ross. Heteroclinic Connections Between Periodic Orbits and Resonance Transitions in Celestial Mechanics. Chaos: An Interdisciplinary Journal of Nonlinear Science, 10: 427, 2000.

[28] W. Koon, M. Lo, J. Marsden, and S. Ross. Low Energy Transfer to the Moon. Celestial Mechanics and Dynamical Astronomy, 81: 63-73, 2001.

[29] M. Lo, B. Williams, W. Bollman, D. Han, Y. Hahn, J. Bell, E. Hirst, R. Corwin, P. Hong, K. Howell, B. Barden, and R. Wilson. Genesis mission design. J. Astron. Sci., 49: 169-184, 2001.

[30] M.W. Lo and M.J. Chung. Lunar Sample Return via the Interplanetary Superhighway. In AIAA/AAS Conference, 886: 100-110, 2002.

[31] L. Mingtao and Z. Jianhua. Impulsive Lunar Halo Transfers Using the Stable Manifolds and Lunar Flybys. Acta Astronautica, 66: 1481-1492, 2010.

[32] G. Mingotti, F. Topputo, and F. Bernelli-Zazzera. Combined Optimal LowThrust and Stable-Manifold Trajectories to the Earth-Moon Halo Orbits. In AIP Conference Proceedings, 886: 100-112, 2007.

[33] M.T. Ozimek and K.C. Howell. Low-thrust transfers in the earth-moon system, including applications to libration point orbits. Journal of Guidance, Control, and Dynamics, 33, 2010.

[34] J.S. Parker and G.H. Born. Direct luna Halo orbit transfers. In $A A S / A I A A$ Spaceflight Dynamics Conference, 2007.

[35] J.S. Parker. Families of Low-Energy Lunar Halo Transfers. In AAS/AIAA Spaceflight Dynamics Conference, 90: 1-20, 2006.

[36] P. Pergola. Low Thrust Transfer to Backflip Orbits. Advances in Space Research, 2010.

[37] C. Simó, G. Gómez, A. Jorba, and J. Masdemont. The Bicircular Model near the Triangular Libration Points of the RTBP. In From Newton to Chaos, 343-370, 1995.

[38] V. Szebehely. Theory of Orbits: the Restricted Problem of Three Bodies. Academic Press New York, 1967.

[39] F. Topputo and E. Belbruno. Computation of the Weak Stability Boundaries: Sun-Jupiter System. Celestial Mechanics and Dynamical Astronomy, 105(1): $3-17,2009$. 
[40] K. Yagasaki. Sun-Perturbed Earth to Moon transfer with low energy and moderate flight time. Celestial Mechanics and Dynamical Astronomy, 90: 197$212,2004$.

[41] K. Yagasaki. Computation of low energy Earth-to-Moon transfers with moderate flight time. Physica, 197: 313-331, 2004. 